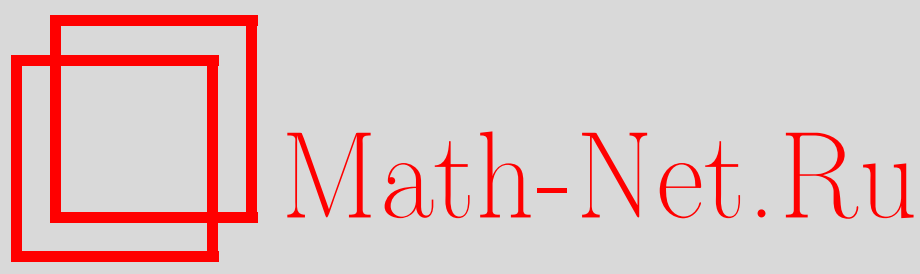

Л. К. Ширяева, О трехпараметрической копула-функции Граббса, Изв. вузов. Матем., 2019, номер 3, 54-71

DOI: https://doi.org/10.26907/0021-3446-2019-3-54-71

Использование Общероссийского математического портала Math-Net.Ru подразумевает, что вы прочитали и согласны с пользовательским соглашением

http: //www.mathnet.ru/rus/agreement

Параметры загрузки:

IP : 52.90 .164 .192

26 апреля 2023 г., 15:43:44

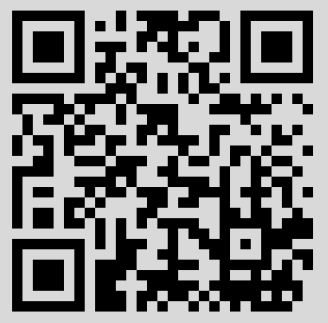


Известия вузов. Математика

2019, № 3, с. 54-71 https://kpfu.ru/science/nauchnye-izdaniya/ivrm/

e-mail: izvuz.matem@kpfu.ru

\title{
Л.К. ШИРЯЕВА
}

\section{О ТРЕХПАРАМЕТРИЧЕСКОЙ КОПУЛА-ФУНКЦИИ ГРАББСА}

\begin{abstract}
Аннотация. Изучаются односторонние статистики Граббса, т. е. экстремальные стьюдентизированные отклонения наблюдений от среднего, вычисляемые по нормально распределенной выборке. Рассматривается случай, когда в выборке имеется одно аномальное наблюдение (выброс), неизвестно какое по номеру. Предполагается, что выброс отличается от остальных наблюдений величиной математического ожидания и дисперсии. Исследуются свойства совместного распределения статистик Граббса. Доказывается существование области, в которой совместная функция распределения статистик Граббса есть линейная функция от маргинальных функций распределения этих статистик. Из совместного распределения статистик Граббса строится трехпараметрическая копула Граббса. Доказывается существование области, в которой копула Граббса совпадает с нижней границей Фреше-Хефдинга. Исследуется влияние параметров копулы на форму этой области.
\end{abstract}

Ключевые слова: односторонняя статистика Граббса, стандартизированные минимум и максимум, выброс, нормальный закон распределения, совместная функция распределения стандартизированных минимума и максимума, копула, нижняя граница Фреше-Хефдинга.

УДК: 519.243

DOI: $10.26907 / 0021-3446-2019-3-54-71$

\section{ВВЕДЕНИЕ}

Копула-функции являются универсальным инструментом для моделирования статистических зависимостей случайных переменных. Поэтому копула-модели применяют для описания совместных распределений случайных переменных. Копула может быть извлечена из известного совместного распределения случайных переменных [1]. Такой способ построения копулы основан на известной теореме Склара. Целью данной статьи является исследование свойств новой копулы, построенной из трехпараметрического совместного распределения односторонних статистик Граббса. Дальнейшее содержание статьи организовано следующим образом. В разделе 1 исследуются свойства совместного распределения односторонних статистик Граббса и доказывается существование области, в которой совместная функция распределения статистик Граббса является линейной функцией от маргинальных функций распределения этих статистик. В разделе 2 из совместного распределения односторонних статистик Граббса посредством инверсии строится трехпараметрическая копула Граббса и доказывается существование области $\Sigma_{n}$, в которой копула совпадает с нижней границей Фреше-Хефдинга. В разделе 3 исследуется влияние параметров копулы на границу области $\Sigma_{n}$. В разделе 4 рассматриваются возможные приложения полученных результатов.

Поступила в редакцию 09.02.2018, после доработки 15.08.2018. Принята к публикации 26.09.2018 


\section{1. О СВОЙСТВАХ СОВМЕСТНОГО ТРЕХПАРАМЕТРИЧЕСКОГО РАСПРЕДЕЛЕНИЯ СТАТИСТИК ГРАББСА}

Пусть $X_{1}, X_{2}, \ldots, X_{n-1}, X_{n}$ - набор из $n$ наблюдений; $X_{(1)} \leq X_{(2)} \leq \cdots \leq X_{(n-1)} \leq X_{(n)}$ - вариационный ряд для него; $X_{(j)}-j$-я порядковая статистика $(j=\overline{1, n})$.

Пусть гипотеза $H_{0}$ состоит в том, что наблюдения $X_{1}, X_{2}, \ldots, X_{n-1}, X_{n}$ являются независимыми случайными величинами с нормальным распределением $N\left(a, \sigma^{2}\right)$, параметры $a$ и $\sigma^{2}$ которого предполагаются неизвестными. В качестве гипотезы $H_{1}$ рассмотрим случай, когда какие-либо $(n-1)$ из $n$ наблюдений имеют одинаковое нормальное $N\left(a, \sigma^{2}\right)$-распределение, а одно из них (неизвестно какое по номеру) имеет нормальное распределение $N\left(a+\alpha \sigma, \nu \sigma^{2}\right)$. Таким образом, аномальное наблюдение (выброс) $X_{\text {out }}$ отличается от остальных наблюдений параметром сдвига $\alpha$ и параметром масштаба $\nu>0$, и номер выброса в выборке неизвестен.

Нулевую и альтернативную гипотезы можно формализовать следующим образом [2]: $H_{0}$ : $(\alpha=0, \nu=1)$ и $H_{1}:(\alpha \neq 0)$ или $(\alpha=0, \nu \neq 1)$.

Для тестирования нормальной выборки на наличие в ней аномальных наблюдений часто используют стьюдентизированные порядковые статистики. $\mathrm{K}$ числу таких статистик относят односторонние статистики Граббса, т. е. экстремальные стьюдентизированные отклонения наблюдений от среднего, вычисляемые по выборке из $n$ наблюдений

$$
T_{n,(1)}=\left(\bar{X}-X_{(1)}\right) / S, \quad T_{n}^{(1)}=\left(X_{(n)}-\bar{X}\right) / S,
$$

где $\bar{X}=\frac{1}{n} \sum_{i=1}^{n} X_{i}$ и $S^{2}=\frac{1}{n-1} \sum_{i=1}^{n}\left(X_{i}-\bar{X}\right)^{2}$.

Статистика $T_{n}^{(1)}$ была предложена Е. Пирсоном и Чандра Секаром для проверки гипотезы о том, является ли наблюдение $X_{(n)}$ аномальным [3]. Ф. Граббс впервые построил таблицу критических точек распределения статистики $T_{n}^{(1)}[4]$.

Известно ([5], с. 168), что статистика $T_{n}^{(1)}$ является статистикой критерия отношения максимальных правдоподобий, применяемого для проверки $H_{0}$ против альтернативы $H_{1}^{\prime}: \alpha>0$, $\nu=1$. На статистике $T_{n,(1)}$ основан критерий отношения максимальных правдоподобий для проверки $H_{0}$ против альтернативы $H_{1}^{\prime \prime}: \alpha<0, \nu=1$ ([5], с. 168).

При справедливости гипотезы $H_{0}$ статистика $T_{n,(1)}$ имеет такой же закон распределения, что и статистика $T_{n}^{(1)}[4]$, т. е.

$$
P\left(T_{n}^{(1)}<t \mid H_{0}\right)=P\left(T_{n,(1)}<t \mid H_{0}\right) \quad \forall t \in \mathbb{R} .
$$

Обозначим $F_{n}^{(1)}(t)=P\left(T_{n}^{(1)}<t \mid H_{0}\right)$. Закон распределения статистики $T_{n}^{(1)}$ (или $\left.T_{n,(1)}\right)$ может быть найден с помощью специальной рекурсивной процедуры, описанной в ([5], с. 115116). В работах ([5], с. 167; [6]) с помощью данной процедуры построены рекурсивные соотношения для функции распределения статистики $T_{n}^{(1)}$ при справедливости гипотезы $H_{0}$

$$
F_{n}^{(1)}(t)=\left\{\begin{array}{l}
0, \quad t \leq \frac{1}{\sqrt{n}}, n \geq 2 ; \\
n \int_{\frac{1}{\sqrt{n}}}^{t} F_{n-1}^{(1)}\left(g_{n}(x)\right) f_{T_{n}}(x) d x, \frac{1}{\sqrt{n}}<t \leq \frac{n-1}{\sqrt{n}}, \quad n \geq 3 ; \\
1, \quad t>\frac{n-1}{\sqrt{n}}, n \geq 2,
\end{array}\right.
$$

где

$$
g_{n}(x)=\frac{n}{n-1} x / \sqrt{\frac{n-1}{n-2}\left(1-\frac{n}{(n-1)^{2}} x^{2}\right)}, \quad|x|<\frac{n-1}{\sqrt{n}}
$$




$$
f_{T_{n}}(x)= \begin{cases}\frac{1}{n-1} \sqrt{\frac{n}{\pi}} \Gamma\left(\frac{n-1}{2}\right) / \Gamma\left(\frac{n-2}{2}\right)\left(1-\frac{n}{(n-1)^{2}} x^{2}\right)^{\frac{n-4}{2}}, & |x|<\frac{n-1}{\sqrt{n}} \\ 0, & |x| \geq \frac{n-1}{\sqrt{n}}\end{cases}
$$

$\Gamma(x)$ - гамма-функция Эйлера.

Обозначим через $\Lambda_{n}\left(t_{1}, t_{2}\right)=P\left(T_{n,(1)}<t_{1}, T_{n}^{(1)}<t_{2} \mid H_{0}\right)$ совместную функцию распределения статистик Граббса. В [7] доказано, что в случае $n>2$ функция $\Lambda_{n}\left(t_{1}, t_{2}\right)$ может быть описана следующим рекурсивным соотношением:

$$
\Lambda_{n}\left(t_{1}, t_{2}\right)=\left\{\begin{array}{l}
F_{n}^{(1)}\left(t_{2}\right), \quad t_{1} \geq \frac{n-1}{\sqrt{n}} ; \quad F_{n}^{(1)}\left(t_{1}\right), \quad t_{2} \geq \frac{n-1}{\sqrt{n}} \\
n \int_{\frac{1}{\sqrt{n}}}^{t_{2}} \Lambda_{n-1}\left(\rho_{n}\left(t_{1},-x\right), g_{n}(x)\right) f_{T_{n}}(x) d x, \quad\left(t_{1}, t_{2}\right) \in \Delta_{n} ; \\
0, \quad\left(t_{1}, t_{2}\right) \notin \Delta_{n}, \quad t_{1}<\frac{n-1}{\sqrt{n}}, \quad t_{2}<\frac{n-1}{\sqrt{n}},
\end{array}\right.
$$

где $F_{n}^{(1)}(t)$ вычисляется по формуле $(1)$;

$$
\rho_{n}(t, z)=\left(t+\frac{z}{n-1}\right) / \sqrt{\frac{n-1}{n-2}\left(1-\frac{n}{(n-1)^{2}} z^{2}\right)}, \quad|z|<\frac{n-1}{\sqrt{n}} ;
$$

функции $g_{n}(x)$ и $f_{T_{n}}(x)$ вычисляются по формулам (2) и (3) соответственно;

$$
\Delta_{n}=\left[1 / \sqrt{n}<t_{1}<(n-1) / \sqrt{n} ; 1 / \sqrt{n}<t_{2}<(n-1) / \sqrt{n}\right] .
$$

В случае $n=2$ имеет место равенство [7]

$$
\Lambda_{2}\left(t_{1}, t_{2}\right)= \begin{cases}1, & \left(t_{1}, t_{2}\right) \in \Delta_{2}, \quad \Delta_{2}=\left[\frac{\sqrt{2}}{2}<t_{1}<\infty ; \frac{\sqrt{2}}{2}<t_{2}<\infty\right] ; \\ 0, & \left(t_{1}, t_{2}\right) \notin \Delta_{2} .\end{cases}
$$

Предположим, что верна альтернативная гипотеза $H_{1}$. Обозначим

$$
\widetilde{T_{n}}=\left(X_{\text {out }}-\bar{X}\right) / S \text {. }
$$

Случайная величина $\widetilde{T_{n}}$ является стьюдентизированным отклонением выброса $X_{\text {out }}$ от среднего, найденным по выборке объема $n$.

Обозначим через $f_{\widetilde{T_{n}}}(\cdot)$ плотность распределения вероятностей случайной величины $\widetilde{T_{n}}$. Закон распределения случайной величины $\widetilde{T_{n}}$ был найден в [8]. В [9] показано, что для вычисления плотности может быть использована функция Эрмита

$$
f_{\widehat{T}_{n}}(t ; \alpha, \nu)=\beta_{n}(t ; \alpha, \nu) \cdot f_{T_{n}}(t),
$$

где

$$
\begin{gathered}
\beta_{n}(t ; \alpha, \nu)=\frac{c_{n}}{q_{n}^{\frac{n-1}{2}}(t)} H_{-n+1}\left(-\frac{t \mu}{\sqrt{2 q_{n}(t)}}\right), \\
c_{n}=\frac{2^{n-1}(n-1)^{n-1}}{\sqrt{\pi} n^{\frac{n-1}{2}}} \cdot \eta^{\frac{n-2}{2}} \cdot e^{-\frac{\mu^{2}}{2}} \cdot \Gamma\left(\frac{n}{2}\right) ; \mu=\alpha \sqrt{\frac{n-1}{1+\nu(n-1)}} ; \eta=\frac{1+\nu(n-1)}{n} ; q_{n}(t)=\eta \frac{(n-1)^{2}}{n}+(1-\eta) t^{2} ; \\
H_{k}(z)=\frac{1}{\Gamma(-k)} \int_{0}^{\infty} e^{-\xi^{2}-2 z \xi} \xi^{-k-1} d \xi-\text { функция Эрмита с отрицательным целым значком } k .
\end{gathered}
$$

Обозначим

$$
\begin{gathered}
G_{n,(1)}(t ; \alpha, \nu)=P\left(T_{n,(1)}<t \mid H_{1}\right), \quad G_{n}^{(1)}(t ; \alpha, \nu)=P\left(T_{n}^{(1)}<t \mid H_{1}\right), \\
\Upsilon_{n}\left(t_{1}, t_{2} ; \alpha, \nu\right)=P\left(T_{n,(1)}<t_{1}, \quad T_{n}^{(1)}<t_{2} \mid H_{1}\right) .
\end{gathered}
$$


В [2] доказано, что для $n>2 \forall \alpha \in \mathbb{R}$ и $\nu>0$ справедливы следующие утверждениия:

$$
\begin{gathered}
G_{n}^{(1)}(t ; \alpha, \nu)=G_{n,(1)}(t ;-\alpha, \nu), \\
G_{n,(1)}(t ; \alpha, \nu)=\left\{\begin{array}{l}
0, \quad 0 \leq t \leq \frac{1}{\sqrt{n}} ; \\
\frac{n-1}{\sqrt{n}} \int_{-t}^{(1)} F_{n-1}\left(\rho_{n}(t,-x)\right) f_{\widetilde{T_{n}}}(x ; \alpha, \nu) d x, \quad \frac{1}{\sqrt{n}}<t<\frac{n-1}{\sqrt{n}} ; \\
1, \quad t \geq \frac{n-1}{\sqrt{n}},
\end{array}\right. \\
\Upsilon_{n}\left(t_{1}, t_{2} ; \alpha, \nu\right)=\left\{\begin{array}{l}
G_{n}^{(1)}\left(t_{2} ; \alpha, \nu\right), \quad t_{1} \geq \frac{n-1}{\sqrt{n}} ; \\
G_{n,(1)}\left(t_{1} ; \alpha, \nu\right), \quad t_{2} \geq \frac{n-1}{\sqrt{n}} ; \\
\int_{2} \Lambda_{n-1}\left(\rho_{n}\left(t_{1},-x\right), \rho_{n}\left(t_{2}, x\right)\right) f_{\widetilde{T_{n}}}(x ; \alpha, \nu) d x, \quad\left(t_{1}, t_{2}\right) \in \Delta_{n} ; \\
-t_{1}\left(t_{1}, t_{2}\right) \notin \Delta_{n}, \quad t_{1}<\frac{n-1}{\sqrt{n}}, t_{2}<\frac{n-1}{\sqrt{n}},
\end{array}\right.
\end{gathered}
$$

где функции распределения $F_{n-1}^{(1)}(\cdot), \Lambda_{n-1}^{(1)}(\cdot)$ и $f_{\widetilde{T_{n}}}(\cdot)$ определены согласно формулам $(1)$, (4) и (7) соответственно, функция $\rho_{n}(\cdot)$ определена согласно формуле $(5)$, область $\Delta_{n}-$ согласно (6).

В случае $n=2$ справедливы равенства

$$
G_{2}^{(1)}(t ; \alpha, \nu)=G_{2,(1)}(t ; \alpha, \nu)=F_{2}^{(1)}(t), \quad \Upsilon_{2}\left(t_{1}, t_{2} ; \alpha, \nu\right)=\Lambda_{2}\left(t_{1}, t_{2}\right) .
$$

В [10] из совместного распределения статистик Граббса $T_{n,(1)}$ и $T_{n}^{(1)}$, найденного для случая справедливости гипотезы $H_{0}$, построена однопараметрическая копула и исследованы ее свойства. В данной работе из совместного распределения $T_{n,(1)}$ и $T_{n}^{(1)}$, найденного для случая справедливости гипотезы $H_{1}$, будет построена трехпараметрическая копула и исследованы ее свойства.

Для этого докажем теоремы, необходимые для исследования совместного распределения статистик $T_{n,(1)}$ и $T_{n}^{(1)}$.

Теорема 1. Пусть гипотеза $H_{0}$ верна и область $\Xi_{n}=\left[\frac{1}{\sqrt{n}} \leq t_{1} \leq \frac{n-1}{\sqrt{n}}, \theta_{n}^{+}\left(t_{1}\right) \leq t_{2} \leq \frac{n-1}{\sqrt{n}}\right]$. Тогда для $n>2$ u $\forall\left(t_{1}, t_{2}\right) \in \Xi_{n}$ совместная функция распределения статистик $T_{n,(1)} u$ $T_{n}^{(1)}$ выражается через их маргинальные функиии распределения по формуле

$$
\Lambda_{n}\left(t_{1}, t_{2}\right)=F_{n}^{(1)}\left(t_{1}\right)+F_{n}^{(1)}\left(t_{2}\right)-1,
$$

где $\theta_{n}^{+}(t)=\frac{t}{n-1}+\sqrt{n-2} \sqrt{1-\frac{n}{(n-1)^{2}} t^{2}}$.

Доказательство. Заметим, что $\Xi_{n} \subseteq \Delta_{n}$. Поэтому $\forall\left(t_{1}, t_{2}\right) \in \Xi_{n}$ формулу (4) можно переписать в виде

$\Lambda_{n}\left(t_{1}, t_{2}\right)=n \int_{\frac{1}{\sqrt{n}}}^{\frac{n-1}{\sqrt{n}}} \Lambda_{n-1}\left(\rho_{n}\left(t_{1},-x\right), g_{n}(x)\right) f_{T_{n}}(x) d x-n \int_{t_{2}}^{\frac{n-1}{\sqrt{n}}} \Lambda_{n-1}\left(\rho_{n}\left(t_{1},-x\right), g_{n}(x)\right) f_{T_{n}}(x) d x$.

Отсюда с учетом (4) получим

$$
\begin{gathered}
\Lambda_{n}\left(t_{1}, t_{2}\right)=\Lambda_{n}\left(t_{1}, \frac{n-1}{\sqrt{n}}\right)-n \int_{t_{2}}^{\frac{n-1}{\sqrt{n}}} \Lambda_{n-1}\left(\rho_{n}\left(t_{1},-x\right), g_{n}(x)\right) f_{T_{n}}(x) d x, \\
\Lambda_{n}\left(t_{1}, t_{2}\right)=F_{n}^{(1)}\left(t_{1}\right)-n \int_{t_{2}}^{\frac{n-1}{\sqrt{n}}} \Lambda_{n-1}\left(\rho_{n}\left(t_{1},-x\right), g_{n}(x)\right) f_{T_{n}}(x) d x .
\end{gathered}
$$


Обозначим $\theta_{n}^{ \pm}(t)=\frac{t}{n-1} \pm \sqrt{n-2} \sqrt{1-\frac{n}{(n-1)^{2}} t^{2}}$. С учетом (5) легко убедиться, что $\forall t \in$ $\left(\frac{1}{\sqrt{n}}, \frac{n-1}{\sqrt{n}}\right]$ справедливо

$$
\rho_{n}\left(t,-\theta_{n}^{ \pm}(t)\right)=\frac{n-2}{\sqrt{n-1}} .
$$

Нетрудно также проверить, что $\forall t \in\left(\frac{1}{\sqrt{n}}, \frac{n-1}{\sqrt{n}}\right]$ функция $\rho_{n}(t,-x)$ является убывающей функцией аргумента $x$, если $x \in\left(-\frac{n-1}{\sqrt{n}}, \frac{n-1}{n t}\right)$, и возрастающей, если $x \in\left(\frac{n-1}{n t}, \frac{n-1}{\sqrt{n}}\right)$. Кроме того, $\forall t \in[1 / \sqrt{n},(n-1) / \sqrt{n})$ справедливо

$$
\sqrt{\frac{(n-2)\left(n t^{2}-1\right)}{n(n-1)}}=\rho_{n}\left(t,-\frac{n-1}{n t}\right)<\frac{n-2}{\sqrt{n-1}} .
$$

Но тогда $\forall t_{1} \in\left[\frac{1}{\sqrt{n}}, \frac{n-1}{\sqrt{n}}\right]$ имеем

$$
\begin{aligned}
& \rho_{n}\left(t_{1},-x\right) \geq \frac{n-2}{\sqrt{n-1}}, \text { если } x \in\left(-\frac{n-1}{\sqrt{n}}, \theta_{n}^{-}\left(t_{1}\right)\right] \cup\left[\theta_{n}^{+}\left(t_{1}\right), \frac{n-1}{\sqrt{n}}\right) ; \\
& \rho_{n}\left(t_{1},-x\right)<\frac{n-2}{\sqrt{n-1}}, \text { если } x \in\left(\theta_{n}^{-}\left(t_{1}\right), \theta_{n}^{+}\left(t_{1}\right)\right) .
\end{aligned}
$$

Следовательно, $\forall\left(t_{1}, t_{2}\right) \in \Xi_{n}$ и $t_{2} \leq x \leq(n-1) / \sqrt{n}$ в силу (4) имеем

$$
\Lambda_{n-1}\left(\rho_{n}\left(t_{1},-x\right), g_{n}(x)\right)=F_{n-1}^{(1)}\left(g_{n}(x)\right) .
$$

Поэтому (13) примет вид

$$
\Lambda_{n}\left(t_{1}, t_{2}\right)=F_{n}^{(1)}\left(t_{1}\right)-n \int_{t_{2}}^{\frac{n-1}{\sqrt{n}}} F_{n-1}^{(1)}\left(g_{n}(x)\right) f_{T_{n}}(x) d x, \text { если }\left(t_{1}, t_{2}\right) \in \Xi_{n} .
$$

Полученное равенство можно представить в виде

$$
\Lambda_{n}\left(t_{1}, t_{2}\right)=F_{n}^{(1)}\left(t_{1}\right)+n \int_{\frac{1}{\sqrt{n}}}^{t_{2}} F_{n-1}^{(1)}\left(g_{n}(x)\right) f_{T_{n}}(x) d x-n \int_{\frac{1}{\sqrt{n}}}^{\frac{n-1}{\sqrt{n}}} F_{n-1}^{(1)}\left(g_{n}(x)\right) f_{T_{n}}(x) d x,
$$

если $\left(t_{1}, t_{2}\right) \in \Xi_{n}$. Воспользовавшись $(1)$, получим

$$
\Lambda_{n}\left(t_{1}, t_{2}\right)=F_{n}^{(1)}\left(t_{1}\right)+F_{n}^{(1)}\left(t_{2}\right)-F_{n}^{(1)}((n-1) / \sqrt{n}), \text { если }\left(t_{1}, t_{2}\right) \in \Xi_{n} .
$$

Учитывая, что $F_{n}^{(1)}((n-1) / \sqrt{n})=1$, равенство $(17) \forall\left(t_{1}, t_{2}\right) \in \Xi_{n}$ примет вид (12). Следствие 1. Пусть $A_{n}=\left[\frac{1}{\sqrt{n}} \leq t_{1} \leq \frac{n-1}{\sqrt{n}}, t_{2} \geq \theta_{n}^{+}\left(t_{1}\right)\right], n>2$. Тогда $\forall\left(t_{1}, t_{2}\right) \in A_{n}$ спра-
ведливо равенство

$$
P\left(\left\{T_{n,(1)} \geq t_{1}\right\} \cap\left\{T_{n}^{(1)} \geq t_{2}\right\} \mid H_{0}\right)=0 .
$$

Доказательство. Пусть гипотеза $H_{0}$ верна. Обозначим

$$
\Xi_{n}^{(0)}=\left[\frac{1}{\sqrt{n}} \leq t_{1} \leq \frac{n-1}{\sqrt{n}}, t_{2}>\frac{n-1}{\sqrt{n}}\right] ; \quad \Xi_{n}=\left[\frac{1}{\sqrt{n}} \leq t_{1} \leq \frac{n-1}{\sqrt{n}}, \theta_{n}^{+}\left(t_{1}\right) \leq t_{2} \leq \frac{n-1}{\sqrt{n}}\right] .
$$

Тогда $A_{n}=\Xi_{n} \cup \Xi_{n}^{(0)}$. Из (1) следует, что $\forall t_{2} \geq \frac{n-1}{\sqrt{n}}$ событие $\left\{T_{n}^{(1)} \geq t_{2}\right\}$ является невозможным. Поэтому

$$
P\left(\left\{T_{n,(1)} \geq t_{1}\right\} \cap\left\{T_{n}^{(1)} \geq t_{2}\right\} \mid H_{0}\right)=0 \forall\left(t_{1}, t_{2}\right) \in \Xi_{n}^{(0)} .
$$


Далее заметим, что для любой двумерной случайной величины $(X, Y)$ и $\forall(a, b) \in \mathbb{R}^{2}$ справедливо

$$
P(\{X \geq a\} \cap\{Y \geq b\})=P(\{X<a\} \cap\{Y<b\})-P(Y<b)-P(X<a)+1 .
$$

Для случайных величин $T_{n,(1)}$ и $T_{n}^{(1)}$ равенство (20) примет вид

$$
P\left(\left\{T_{n,(1)} \geq t_{1}\right\} \cap\left\{T_{n}^{(1)} \geq t_{2}\right\} \mid H_{0}\right)=\Lambda_{n}\left(t_{1}, t_{2}\right)-F_{n}^{(1)}\left(t_{1}\right)-F_{n}^{(1)}\left(t_{2}\right)+1 .
$$

Применив (12) $\forall\left(t_{1}, t_{2}\right) \in \Xi_{n}$, получим

$$
P\left(\left\{T_{n,(1)} \geq t_{1}\right\} \cap\left\{T_{n}^{(1)} \geq t_{2}\right\} \mid H_{0}\right)=0 .
$$

Объединяя (19) и $(21), \forall\left(t_{1}, t_{2}\right) \in A_{n}$ получим (18).

Для исследования свойств совместного распределения статистик $T_{n,(1)}$ и $T_{n}^{(1)}$ в случае, когда верна гипотеза $H_{1}$, доказывается

Лемма 1. Пусть

$$
\begin{gathered}
\theta_{n}^{ \pm}(t)=\frac{t}{n-1} \pm \sqrt{n-2} \sqrt{1-\frac{n}{(n-1)^{2}} t^{2}}, \\
B_{n}=\left[\frac{1}{\sqrt{n}} \leq t \leq \frac{n-1}{\sqrt{n}}, \theta_{n}^{-}(t) \leq x \leq \theta_{n}^{+}(t)\right] .
\end{gathered}
$$

Тогда для $n>2$ u $\forall(t, x) \in B_{n}$ справедливо утверждение

$$
\rho_{n}\left(\theta_{n}^{+}(t), x\right) \geq \theta_{n-1}^{+}\left(\rho_{n}(t,-x)\right) .
$$

Доказательство. Обозначим $h_{n}(\xi)=\sqrt{1-\frac{n}{(n-1)^{2}} \xi^{2}}$, где $|\xi| \leq \frac{n-1}{\sqrt{n}}$.

Используя (5) и (22) неравенство (23) нетрудно привести к виду

$$
U_{n}(t)+\frac{x}{n-2} \geq \frac{\sqrt{(n-1)(n-3)}}{n-2} \sqrt{(n-2) h_{n}^{2}(x)-\left(t-\frac{x}{n-1}\right)^{2}},
$$

где $U_{n}(t)=\sqrt{n-2} h_{n}(t)-\frac{t}{(n-1)(n-2)}$.

Легко проверить, что $\forall(t, x) \in B_{n}$ левая часть неравенства $(24)$ неотрицательна.

Действительно, $\forall(t, x) \in B_{n}$ можно записать $U_{n}(t)+\frac{x}{n-2} \geq U_{n}(t)+\frac{\theta_{n}^{-}(t)}{n-2}$. С учетом (22) получим $U_{n}(t)+\frac{\theta_{n}^{-}(t)}{n-2}=\frac{n-3}{\sqrt{n-2}} h_{n}(t) \geq 0 \quad \forall(t, x) \in B_{n}$. Следовательно, $U_{n}(t)+\frac{x}{n-2} \geq 0$ $\forall(t, x) \in B_{n}$. Возведем в квадрат обе части неравенства $(24)$. После несложных преобразований $\forall(t, x) \in B_{n}$ получим

$$
x^{2}+2 x\left(\frac{h_{n}(t)}{\sqrt{n-2}}-\frac{t}{n-1}\right) \geq \frac{(n-1)(n-3)}{n-2}-\frac{(n-1)(n-3)}{(n-2)^{2}} t^{2}-U_{n}^{2}(t) .
$$

Выделив в левой части (25) полный квадрат, $\forall(t, x) \in B_{n}$ имеем

$$
\left(x+\frac{h_{n}(t)}{\sqrt{n-2}}-\frac{t}{n-1}\right)^{2} \geq S_{n}(t),
$$

где $S_{n}(t)=\frac{(n-1)(n-3)}{n-2}-\frac{(n-1)(n-3)}{(n-2)^{2}} t^{2}-U_{n}^{2}(t)+\left(\frac{h_{n}(t)}{\sqrt{n-2}}-\frac{t}{n-1}\right)^{2}$. 
Выполнив несложные преобразования, нетрудно проверить, что для $|t| \leq \frac{n-1}{\sqrt{n}}$ имеем $S_{n}(t)=0$. Поэтому неравенство $(26) \forall(t, x) \in B_{n}$ примет вид

$$
\left(x+\frac{h_{n}(t)}{\sqrt{n-2}}-\frac{t}{n-1}\right)^{2} \geq 0 .
$$

Таким образом, $\forall(t, x) \in B_{n}$ неравенство (27) равносильно (23).

Свойства совместного распределения односторонних статистик Граббса, необходимые в дальнейшем, содержит

Лемма 2. Пусть $\Xi_{n}=\left[\frac{1}{\sqrt{n}} \leq t_{1} \leq \frac{n-1}{\sqrt{n}}, \theta_{n}^{+}\left(t_{1}\right) \leq t_{2} \leq \frac{n-1}{\sqrt{n}}\right], n>2$. Тогда для $\forall\left(t_{1}, t_{2}\right) \in \Xi_{n}$ $u \forall x \in\left[\theta_{n}^{-}\left(t_{1}\right), \theta_{n}^{+}\left(t_{1}\right)\right]$ справедливо равенство

$$
P\left(\left\{T_{n-1,(1)} \geq \rho_{n}\left(t_{1},-x\right)\right\} \cap\left\{T_{n-1}^{(1)} \geq \rho_{n}\left(t_{2}, x\right)\right\} \mid H_{0}\right)=0,
$$

где функиии $\theta_{n}^{ \pm}(\cdot)$ и $\rho_{n}(\cdot)$ вычисляются по формулам (22) и (5).

Доказательство. Заметим, что $\forall t_{1} \in[1 / \sqrt{n},(n-1) / \sqrt{n}]$ функция $y=\rho_{n}\left(t_{1},-x\right)$ убывает по аргументу $x$, если $-\frac{n-1}{\sqrt{n}}<x<x_{\min }\left(t_{1}\right)=\frac{n-1}{n t_{1}}$, и возрастает, если $x_{\min }\left(t_{1}\right) \leq x<(n-1) / \sqrt{n}$. Поэтому $\forall t_{1} \in[1 / \sqrt{n},(n-1) / \sqrt{n}]$ можно записать

$$
\rho_{n}\left(t_{1},-x\right) \geq \sqrt{\frac{n-2}{n(n-1)}\left(n t_{1}^{2}-1\right)}=\rho_{n}\left(t_{1},-x_{\min }\left(t_{1}\right)\right) .
$$

Тогда $\forall x \in\left[\theta_{n}^{-}\left(t_{1}\right), \theta_{n}^{+}\left(t_{1}\right)\right]$ будет выполняться условие

$$
\sqrt{\frac{n-2}{n(n-1)}\left(n t_{1}^{2}-1\right)} \leq \rho_{n}\left(t_{1},-x\right) \leq \frac{n-2}{\sqrt{n-1}}, \quad \text { если } t_{1} \in\left[\frac{1}{\sqrt{n}}, \frac{n-1}{\sqrt{n}}\right]
$$

(здесь функции $\theta_{n}^{ \pm}(\cdot)$ вычисляются согласно (22) и также учтено условие (14)).

Обозначим $t_{n}^{0}=\sqrt{\frac{2(n-1)}{n(n-2)}}$. Положим

$$
\psi_{n}^{ \pm}(t)=\frac{n-2}{2} t \pm \frac{\sqrt{2(n-1)-n(n-2) t^{2}}}{2}, \text { если } \frac{1}{\sqrt{n}} \leq t \leq t_{n}^{0} .
$$

Несложно убедиться в справедливости утверждений

$$
\begin{gathered}
\rho_{n}\left(t_{1},-\psi_{n}^{-}\left(t_{1}\right)\right)=1 / \sqrt{n-1}, \text { если } t_{1} \in\left[1 / \sqrt{n}, t_{n}^{0}\right], \\
\rho_{n}\left(t_{1},-\psi_{n}^{+}\left(t_{1}\right)\right)=1 / \sqrt{n-1}, \quad \text { если } t_{1} \in\left(1 / \sqrt{n}, t_{n}^{0}\right] \text { и } \lim _{t_{1} \rightarrow \frac{1}{\sqrt{n}}+0} \rho_{n}\left(1 / \sqrt{n},-\psi_{n}^{+}\left(t_{1}\right)\right)=0 .
\end{gathered}
$$

Но тогда для $t_{1} \in\left[1 / \sqrt{n}, t_{n}^{0}\right]$ условие (29) примет вид

$$
0 \leq \rho_{n}\left(t_{1},-x\right)<1 / \sqrt{n-1}, \text { если } x \in\left(\psi_{n}^{-}\left(t_{1}\right), \psi_{n}^{+}\left(t_{1}\right)\right) ;
$$

$1 / \sqrt{n-1} \leq \rho_{n}\left(t_{1},-x\right) \leq(n-2) / \sqrt{n-1}$, если $x \in\left[\theta_{n}^{-}\left(t_{1}\right), \psi_{n}^{-}\left(t_{1}\right)\right] \cup\left[\psi_{n}^{+}\left(t_{1}\right), \theta_{n}^{+}\left(t_{1}\right)\right]$,

а для $t_{1} \in\left(t_{n}^{0},(n-1) / \sqrt{n}\right]-$ вид

$$
1 / \sqrt{n-1}<\rho_{n}\left(t_{1},-x\right) \leq(n-2) / \sqrt{n-1}, \text { если } x \in\left[\theta_{n}^{-}\left(t_{1}\right), \theta_{n}^{+}\left(t_{1}\right)\right] .
$$

Обозначим

$$
\begin{gathered}
C_{n}^{(0)}=\left[t_{n}^{0}<t_{1} \leq(n-1) / \sqrt{n}, \theta_{n}^{-}\left(t_{1}\right) \leq x \leq \theta_{n}^{+}\left(t_{1}\right)\right] \\
C_{n}^{(1)}=\left[1 / \sqrt{n} \leq t_{1} \leq t_{n}^{0}, \theta_{n}^{-}\left(t_{1}\right) \leq x \leq \psi_{n}^{-}\left(t_{1}\right)\right] \cup\left[1 / \sqrt{n} \leq t_{1} \leq t_{n}^{0}, \psi_{n}^{+}\left(t_{1}\right) \leq x \leq \theta_{n}^{+}\left(t_{1}\right)\right] .
\end{gathered}
$$


Пусть $C_{n}=C_{n}^{(0)} \cup C_{n}^{(1)}$. С учетом (32), (33) можно утверждать, что $\forall\left(t_{1}, x\right) \in C_{n}$ выполняется неравенство

$$
1 / \sqrt{n-1} \leq \rho_{n}\left(t_{1},-x\right) \leq(n-2) / \sqrt{n-1} .
$$

Заметим, что $C_{n} \subseteq B_{n}$, где $B_{n}=\left[1 / \sqrt{n} \leq t_{1} \leq(n-1) / \sqrt{n}, \theta_{n}^{-}\left(t_{1}\right) \leq x \leq \theta_{n}^{+}\left(t_{1}\right)\right]$. Тогда согласно лемме 1 имеем

$$
\rho_{n}\left(\theta_{n}^{+}\left(t_{1}\right), x\right) \geq \theta_{n-1}^{+}\left(\rho_{n}\left(t_{1},-x\right)\right), \text { если }\left(t_{1}, x\right) \in C_{n} .
$$

Учитывая, что функция $\rho_{n}(t, x)$ возрастает по аргументу $t$, для $t_{2} \geq \theta_{n}^{+}\left(t_{1}\right)$ можно записать

$$
\rho_{n}\left(t_{2}, x\right) \geq \rho_{n}\left(\theta_{n}^{+}\left(t_{1}\right), x\right) .
$$

Следовательно, для $\left(t_{1}, x\right) \in C_{n}$ и $t_{2} \geq \theta_{n}^{+}\left(t_{1}\right)$ получим

$$
\rho_{n}\left(t_{2}, x\right) \geq \theta_{n-1}^{+}\left(\rho_{n}\left(t_{1},-x\right)\right) .
$$

Обозначим $A_{n-1}=\left[1 / \sqrt{n-1} \leq \xi_{1} \leq(n-2) / \sqrt{n-1}, \xi_{2} \geq \theta_{n-1}^{+}\left(\xi_{1}\right)\right]$.

Положим $\xi_{1}=\rho_{n}\left(t_{1},-x\right), \xi_{2}=\rho_{n}\left(t_{2}, x\right)$. Из условий $(34),(35)$ следует, что для $\left(t_{1}, x\right) \in C_{n}$ и $t_{2} \geq \theta_{n}^{+}\left(t_{1}\right)$ точка $M\left(\xi_{1}, \xi_{2}\right) \in A_{n-1}$. Тогда, применив следствие 1 , для $\left(t_{1}, x\right) \in C_{n}$ и $t_{2} \in\left[\theta_{n}^{+}\left(t_{1}\right), \frac{n-1}{\sqrt{n}}\right]$ получим

$$
P\left(\left\{T_{n-1,(1)} \geq \rho_{n}\left(t_{1},-x\right)\right\} \cap\left\{T_{n-1}^{(1)} \geq \rho_{n}\left(t_{2}, x\right)\right\} \mid H_{0}\right)=0 .
$$

Введем следующие обозначения: $D_{n}=\left[1 / \sqrt{n} \leq t_{1} \leq t_{n}^{0}, \psi_{n}^{-}\left(t_{1}\right)<x<\psi_{n}^{+}\left(t_{1}\right)\right]$. Заметим, что из (1) вытекает, что область возможных значений статистики $T_{n-1,(1)}$ можно описать неравенством $1 / \sqrt{n-1} \leq T_{n-1,(1)} \leq(n-2) / \sqrt{n-1}$. Учитывая условие $(31), \forall\left(t_{1}, x\right) \in D_{n}$ получим $P\left(\left\{T_{n-1,(1)} \geq \rho_{n}\left(t_{1},-x\right)\right\} \cap\left\{T_{n-1}^{(1)} \geq \rho_{n}\left(t_{2}, x\right)\right\} \mid H_{0}\right)=P\left(\left\{T_{n-1}^{(1)} \geq \rho_{n}\left(t_{2}, x\right)\right\} \mid H_{0}\right)$. Осталось доказать, что $\forall\left(t_{1}, x\right) \in D_{n}$ и $t_{2} \in\left[\theta_{n}^{+}\left(t_{1}\right),(n-1) / \sqrt{n}\right]$ справедливо утверждение

$$
P\left(\left\{T_{n-1}^{(1)} \geq \rho_{n}\left(t_{2}, x\right)\right\} \mid H_{0}\right)=0 .
$$

Для этого заметим, что в силу $(5)$ функция $y=\rho_{n}(t, x)$ возрастает по аргументу $t$. Поэтому

$$
\rho_{n}\left(t_{2}, x\right) \geq \rho_{n}\left(\theta_{n}^{+}\left(t_{1}\right), x\right), \text { если }\left(t_{1}, t_{2}\right) \in \Xi_{n} \quad \text { и }|x|<(n-1) / \sqrt{n} .
$$

Кроме того, функция $y=\rho_{n}\left(\theta_{n}^{+}\left(t_{1}\right), x\right)$ возрастает по аргументу $x$, если $-\frac{n-1}{n \theta_{n}^{+}\left(t_{1}\right)}=z_{\min }\left(t_{1}\right) \leq$ $x<\frac{n-1}{\sqrt{n}}$. Также легко убедиться в справедливости утверждения

$$
\left(\psi_{n}^{-}\left(t_{1}\right), \psi_{n}^{+}\left(t_{1}\right)\right) \subseteq\left[z_{\min }\left(t_{1}\right),(n-1) / \sqrt{n}\right], \text { если } t_{1} \in\left[1 / \sqrt{n}, t_{n}^{0}\right] .
$$

Поэтому функция $y=\rho_{n}\left(\theta_{n}^{+}\left(t_{1}\right), x\right)$ возрастает по аргументу $x$, если $\psi_{n}^{-}\left(t_{1}\right)<x<\psi_{n}^{+}\left(t_{1}\right)$. Тогда $\forall\left(t_{1}, x\right) \in D_{n}$ можно записать $\rho_{n}\left(\theta_{n}^{+}\left(t_{1}\right), x\right)>\rho_{n}\left(\theta_{n}^{+}\left(t_{1}\right), \psi_{n}^{-}\left(t_{1}\right)\right)$. Следовательно,

$$
\rho_{n}\left(t_{2}, x\right) \geq \rho_{n}\left(\theta_{n}^{+}\left(t_{1}\right), \psi_{n}^{-}\left(t_{1}\right)\right), \text { если }\left(t_{1}, x\right) \in D_{n} \text { и } t_{2} \in\left[\theta_{n}^{+}\left(t_{1}\right),(n-1) / \sqrt{n}\right] .
$$

Используя формулы (5), (22) и (30), нетрудно проверить справедливость утверждения

$$
\rho_{n}\left(\theta_{n}^{+}\left(t_{1}\right), \psi_{n}^{-}\left(t_{1}\right)\right) \geq(n-2) / \sqrt{n-1}, \text { если } t_{1} \in\left[1 / \sqrt{n}, t_{n}^{0}\right] .
$$

Поэтому для $\left(t_{1}, x\right) \in D_{n}$ и $t_{2} \in\left[\theta_{n}^{+}\left(t_{1}\right),(n-1) / \sqrt{n}\right]$ условие $(38)$ примет вид

$$
\rho_{n}\left(t_{2}, x\right) \geq(n-2) / \sqrt{n-1} .
$$

Теперь заметим, что из соотношений (1) следует справедливость утверждения

$$
P\left(T_{n-1}^{(1)}>\xi \mid H_{0}\right)=0, \text { если } \xi \geq(n-2) / \sqrt{n-1} .
$$


С учетом (39) и (40) для $\left(t_{1}, x\right) \in D_{n}$ и $t_{2} \in\left[\theta_{n}^{+}\left(t_{1}\right), \frac{n-1}{\sqrt{n}}\right]$ получим (37). Следовательно, для $\left(t_{1}, x\right) \in D_{n}$ и $t_{2} \in\left[\theta_{n}^{+}\left(t_{1}\right),(n-1) / \sqrt{n}\right]$ имеем

$$
P\left(\left\{T_{n-1,(1)} \geq \rho_{n}\left(t_{1},-x\right)\right\} \cap\left\{T_{n-1}^{(1)} \geq \rho_{n}\left(t_{2}, x\right)\right\} \mid H_{0}\right)=0 .
$$

Объединяя (36) и (41) получим, что $\forall\left(t_{1}, t_{2}\right) \in \Xi_{n}$ и $\forall x \in\left[\theta_{n}^{-}\left(t_{1}\right), \theta_{n}^{+}\left(t_{1}\right)\right]$ имеет место равенство (28).

Теорема 2. Пусть гипотеза $H_{1}$ верна и область $\Xi_{n}=\left[\frac{1}{\sqrt{n}} \leq t_{1} \leq \frac{n-1}{\sqrt{n}}, \theta_{n}^{+}\left(t_{1}\right) \leq t_{2} \leq \frac{n-1}{\sqrt{n}}\right]$. Тогда для $n>2$ u $\forall\left(t_{1}, t_{2}\right) \in \Xi_{n}$ совместная функиия распределения статистик $T_{n,(1)} u$ $T_{n}^{(1)}$ выражается через их маргинальные функции распределения по формуле

$$
\Upsilon_{n}\left(t_{1}, t_{2} ; \alpha, \nu\right)=G_{n,(1)}\left(t_{1} ; \alpha, \nu\right)+G_{n}^{(1)}\left(t_{2} ; \alpha, \nu\right)-1,
$$

где $\theta_{n}^{+}(t)=\frac{t}{n-1}+\sqrt{n-2} \sqrt{1-\frac{n}{(n-1)^{2}} t^{2}}$.

Доказательство. Пусть гипотеза $H_{1}$ верна. Применим равенство (20) для случайных величин $T_{n,(1)}$ и $T_{n}^{(1)}$. В результате получим

$$
J_{n}\left(t_{1}, t_{2}\right)=\Upsilon_{n}\left(t_{1}, t_{2} ; \alpha, \nu\right)-G_{n,(1)}\left(t_{1} ; \alpha, \nu\right)-G_{n}^{(1)}\left(t_{2} ; \alpha, \nu\right)+1,
$$

где $J_{n}\left(t_{1}, t_{2}\right)=P\left(\left\{T_{n,(1)} \geq t_{1}\right\} \cap\left\{T_{n}^{(1)} \geq t_{2}\right\} \mid H_{1}\right)$. Остается доказать, что $\forall\left(t_{1}, t_{2}\right) \in \Xi_{n}$ справедливо

$$
J_{n}\left(t_{1}, t_{2}\right)=0 .
$$

Для этого заметим, что $\Xi_{n} \subseteq \Delta_{n}$. Используя формулы $(9)-(11), \quad \forall\left(t_{1}, t_{2}\right) \in \Xi_{n} \subseteq \Delta_{n}$ равенство (43) можно привести к виду

$$
\begin{gathered}
J_{n}\left(t_{1}, t_{2}\right)=\int_{-t_{1}}^{t_{2}} \Lambda_{n-1}\left(\rho_{n}\left(t_{1},-x\right), \rho_{n}\left(t_{2}, x\right)\right) f_{\widetilde{T_{n}}}(x ; \alpha, \nu) d x- \\
-\int_{-t_{1}}^{\frac{n-1}{\sqrt{n}}} F_{n-1}^{(1)}\left(\rho_{n}\left(t_{1},-x\right)\right) f_{\widetilde{T_{n}}}(x ; \alpha, \nu) d x- \\
\quad-\int_{-\frac{n-1}{\sqrt{n}}}^{t_{2}} F_{n-1}^{(1)}\left(\rho_{n}\left(t_{2}, x\right)\right) f_{\widetilde{T_{n}}}(x ; \alpha, \nu) d x+1 .
\end{gathered}
$$

Здесь также учтено, что в силу (7), (8) для $|x| \leq(n-1) / \sqrt{n}$ имеем

$$
f_{\widetilde{T_{n}}}(x ;-\alpha, \nu)=f_{\widetilde{T_{n}}}(-x ; \alpha, \nu) .
$$

Первое слагаемое в (45) можно переписать следующим образом:

$$
\begin{aligned}
& \int_{-t_{1}}^{t_{2}} \Lambda_{n-1}\left(\rho_{n}\left(t_{1},-x\right), \rho_{n}\left(t_{2}, x\right)\right) f_{\widetilde{T_{n}}}(x ; \alpha, \nu) d x=\int_{-\frac{n-1}{\sqrt{n}}}^{\frac{n-1}{\sqrt{n}}} \Lambda_{n-1}\left(\rho_{n}\left(t_{1},-x\right), \rho_{n}\left(t_{2}, x\right)\right) f_{\widetilde{T_{n}}}(x ; \alpha, \nu) d x- \\
- & \int_{-\frac{n-1}{\sqrt{n}}}^{-t_{1}} \Lambda_{n-1}\left(\rho_{n}\left(t_{1},-x\right), \rho_{n}\left(t_{2}, x\right)\right) f_{\widetilde{T_{n}}}(x ; \alpha, \nu) d x-\int_{t_{2}}^{\frac{n-1}{\sqrt{n}}} \Lambda_{n-1}\left(\rho_{n}\left(t_{1},-x\right), \rho_{n}\left(t_{2}, x\right)\right) f_{\widetilde{T_{n}}}(x ; \alpha, \nu) d x .
\end{aligned}
$$

Учитывая $(15), \forall\left(t_{1}, t_{2}\right) \in \Xi_{n}$ можно записать

$$
\rho_{n}\left(t_{1},-x\right) \geq \frac{n-2}{\sqrt{n-1}}, \text { если } t_{2} \leq x<\frac{n-1}{\sqrt{n}} .
$$


Применим формулу (4) с учетом условия (48). В результате $\forall\left(t_{1}, t_{2}\right) \in \Xi_{n}$ получим

$$
\begin{gathered}
\Lambda_{n-1}\left(\rho_{n}\left(t_{1},-x\right), \rho_{n}\left(t_{2}, x\right)\right)=F_{n-1}^{(1)}\left(\rho_{n}\left(t_{2}, x\right)\right), \quad \text { если } x \in\left[t_{2}, \frac{n-1}{\sqrt{n}}\right) ; \\
\int_{t_{2}}^{\frac{n-1}{\sqrt{n}}} \Lambda_{n-1}\left(\rho_{n}\left(t_{1},-x\right), \rho_{n}\left(t_{2}, x\right)\right) f_{\widetilde{T_{n}}}(x ; \alpha, \nu) d x=\int_{t_{2}}^{\frac{n-1}{\sqrt{n}}} F_{n-1}^{(1)}\left(\rho_{n}\left(t_{2}, x\right)\right) f_{\widetilde{T_{n}}}(x ; \alpha, \nu) d x .
\end{gathered}
$$

Аналогично можно убедиться, что $\forall\left(t_{1}, t_{2}\right) \in \Xi_{n}$ справедливо

$$
\int_{-\frac{n-1}{\sqrt{n}}}^{-t_{1}} \Lambda_{n-1}\left(\rho_{n}\left(t_{1},-x\right), \rho_{n}\left(t_{2}, x\right)\right) f_{\widetilde{T}_{n}}(x ; \alpha, \nu) d x=\int_{-\frac{n-1}{\sqrt{n}}}^{-t_{1}} F_{n-1}^{(1)}\left(\rho_{n}\left(t_{1},-x\right)\right) f_{\widetilde{T}_{n}}(x ; \alpha, \nu) d x .
$$

С учетом (49), (50) $\forall\left(t_{1}, t_{2}\right) \in \Xi_{n}$ формула (47) примет вид

$$
\begin{array}{r}
\int_{-t_{1}}^{t_{2}} \Lambda_{n-1}\left(\rho_{n}\left(t_{1},-x\right), \rho_{n}\left(t_{2}, x\right)\right) f_{\widetilde{T_{n}}}(x ; \alpha, \nu) d x=\int_{-\frac{n-1}{\sqrt{n}}}^{\frac{n-1}{\sqrt{n}}} \Lambda_{n-1}\left(\rho_{n}\left(t_{1},-x\right), \rho_{n}\left(t_{2}, x\right)\right) f_{\widetilde{T_{n}}}(x ; \alpha, \nu) d x- \\
-\int_{-\frac{n-1}{\sqrt{n}}}^{-t_{1}} F_{n-1}^{(1)}\left(\rho_{n}\left(t_{1},-x\right)\right) f_{\widetilde{T}_{n}}(x ; \alpha, \nu) d x-\int_{t_{2}}^{\frac{n-1}{\sqrt{n}}} F_{n-1}^{(1)}\left(\rho_{n}\left(t_{2}, x\right)\right) f_{\widetilde{T_{n}}}(x ; \alpha, \nu) d x .
\end{array}
$$

Воспользовавшись последним равенством, $\forall\left(t_{1}, t_{2}\right) \in \Xi_{n}$ формулу (45) приведем к виду

$$
J_{n}\left(t_{1}, t_{2}\right)=\int_{-\frac{n-1}{\sqrt{n}}}^{\frac{n-1}{\sqrt{n}}} Q_{n}\left(t_{1}, t_{2}, x\right) f_{\widehat{T}_{n}}(x ; \alpha, \nu) d x
$$

где $Q_{n}\left(t_{1}, t_{2}, x\right)=\Lambda_{n-1}\left(\rho_{n}\left(t_{1},-x\right), \rho_{n}\left(t_{2}, x\right)\right)-F_{n-1}^{(1)}\left(\rho_{n}\left(t_{1},-x\right)\right)-F_{n-1}^{(1)}\left(\rho_{n}\left(t_{2}, x\right)\right)+1$.

Здесь также учтено, что в силу $(3),(7)$ для плотности распределения вероятностей случайной величины $\widetilde{T_{n}}$ имеет место равенство

$$
\int_{-\frac{n-1}{\sqrt{n}}}^{\frac{n-1}{\sqrt{n}}} f_{\widetilde{T_{n}}}(x ; \alpha, \nu) d x=1 .
$$

Для проверки справедливости утверждения (44) преобразуем множитель $Q_{n}\left(t_{1}, t_{2}, x\right)$, присутствующий в подинтегральном выражении для $J_{n}\left(t_{1}, t_{2}\right)$ в $(51)$. Для этого заметим, что с учетом обозначений функций $\Lambda_{n}(\cdot)$ и $F_{n}^{(1)}(\cdot)$ формулу для $Q_{n}\left(t_{1}, t_{2}, x\right)$ можно переписать в виде

$$
\begin{aligned}
& Q_{n}\left(t_{1}, t_{2}, x\right)=P\left(\left\{T_{n-1,(1)}<\rho_{n}\left(t_{1},-x\right)\right\} \cap\left\{T_{n-1}^{(1)}<\rho_{n}\left(t_{2}, x\right)\right\} \mid H_{0}\right)- \\
& \quad-P\left(\left\{T_{n-1,(1)}<\rho_{n}\left(t_{1},-x\right)\right\} \mid H_{0}\right)-P\left(\left\{T_{n-1}^{(1)}<\rho_{n}\left(t_{2}, x\right)\right\} \mid H_{0}\right)+1 .
\end{aligned}
$$

Отсюда с учетом (20) получим

$$
Q_{n}\left(t_{1}, t_{2}, x\right)=P\left(\left\{T_{n-1,(1)} \geq \rho_{n}\left(t_{1},-x\right)\right\} \cap\left\{T_{n-1}^{(1)} \geq \rho_{n}\left(t_{2}, x\right)\right\} \mid H_{0}\right) .
$$

Следовательно, $\forall\left(t_{1}, t_{2}\right) \in \Xi_{n}$ равенство (51) примет вид

$$
J_{n}\left(t_{1}, t_{2}\right)=\int_{-\frac{n-1}{\sqrt{n}}}^{\frac{n-1}{\sqrt{n}}} P\left(\left\{T_{n-1,(1)} \geq \rho_{n}\left(t_{1},-x\right)\right\} \cap\left\{T_{n-1}^{(1)} \geq \rho_{n}\left(t_{2}, x\right)\right\} \mid H_{0}\right) f_{\widetilde{T}_{n}}(x ; \alpha, \nu) d x .
$$


Теперь заметим, что в силу условия (15) $\forall t_{1} \in[1 / \sqrt{n},(n-1) / \sqrt{n}]$ и $\forall x \in\left(-(n-1) / \sqrt{n}, \theta_{n}^{-}\left(t_{1}\right)\right]$ $\cup\left[\theta_{n}^{+}\left(t_{1}\right),(n-1) / \sqrt{n}\right)$ можно применить $(40)$, положив $\xi=\rho_{n}\left(t_{1},-x\right)$. В результате $\forall t_{1} \in\left[\frac{1}{\sqrt{n}}, \frac{n-1}{\sqrt{n}}\right]$ получим

$$
P\left(\left\{T_{n-1,(1)}>\rho_{n}\left(t_{1},-x\right)\right\} \mid H_{0}\right)=0, \text { если } x \in\left(-(n-1) / \sqrt{n}, \theta_{n}^{-}\left(t_{1}\right)\right] \cup\left[\theta_{n}^{+}\left(t_{1}\right),(n-1) / \sqrt{n}\right) .
$$

Поэтому $\forall\left(t_{1}, t_{2}\right) \in \Xi_{n}$ и $\forall x \in\left(-(n-1) / \sqrt{n}, \theta_{n}^{-}\left(t_{1}\right)\right] \cup\left[\theta_{n}^{+}\left(t_{1}\right),(n-1) / \sqrt{n}\right)$ имеем

$$
P\left(\left\{T_{n-1,(1)} \geq \rho_{n}\left(t_{1},-x\right)\right\} \cap\left\{T_{n-1}^{(1)} \geq \rho_{n}\left(t_{2}, x\right)\right\} \mid H_{0}\right)=0 .
$$

Следовательно, $\forall\left(t_{1}, t_{2}\right) \in \Xi_{n}$ формула (53) примет вид

$$
J_{n}\left(t_{1}, t_{2}\right)=\int_{\theta_{n}^{-}\left(t_{1}\right)}^{\theta_{n}^{+}\left(t_{1}\right)} P\left(\left\{T_{n-1,(1)} \geq \rho_{n}\left(t_{1},-x\right)\right\} \cap\left\{T_{n-1}^{(1)} \geq \rho_{n}\left(t_{2}, x\right)\right\} \mid H_{0}\right) f_{\widetilde{T}_{n}}(x ; \alpha, \nu) d x .
$$

Применив лемму 2, получим, что $\forall\left(t_{1}, t_{2}\right) \in \Xi_{n}$ и $\forall x \in\left[\theta_{n}^{-}\left(t_{1}\right), \theta_{n}^{+}\left(t_{1}\right)\right]$ справедливо утверждение $P\left(\left\{T_{n-1,(1)} \geq \rho_{n}\left(t_{1},-x\right)\right\} \cap\left\{T_{n-1}^{(1)} \geq \rho_{n}\left(t_{2}, x\right)\right\} \mid H_{0}\right)=0$. Тогда формула (54) примет вид (44). Следовательно, $\forall\left(t_{1}, t_{2}\right) \in \Xi_{n}$ равенство (43) имеет вид

$$
0=\Upsilon_{n}\left(t_{1}, t_{2} ; \alpha, \nu\right)-G_{n,(1)}\left(t_{1} ; \alpha, \nu\right)-G_{n}^{(1)}\left(t_{2} ; \alpha, \nu\right)+1,
$$

откуда и вытекает справедливость (42).

\section{2. ИССЛЕДОВАНИЕ СВОЙСТВ ТРЕХПАРАМЕТРИЧЕСКОЙ КОПУЛЫ ГРАББСА}

Трехпараметрическую копулу Граббса получим инверсией из двумерного распределения $\Upsilon_{n}\left(t_{1}, t_{2} ; \alpha, \nu\right)$, используя следствие из теоремы Склара ([11], с. 18; [1]).

Обозначим через $\phi_{n,(1)}(\cdot), \phi_{n}^{(1)}(\cdot)$ функции, обратные функциям частных распределений $G_{n,(1)}$ и $G_{n}^{(1)}$, так что $\forall \xi \in[0,1]$ и $\forall t \in[1 / \sqrt{n},(n-1) / \sqrt{n}]$ справедливы утверждения

$$
\begin{array}{cl}
G_{n,(1)}\left(\phi_{n,(1)}(\xi ; \alpha, \nu) ; \alpha, \nu\right) & =\xi, \quad \phi_{n,(1)}\left(G_{n,(1)}(t ; \alpha, \nu) ; \alpha, \nu\right)=t, \\
G_{n}^{(1)}\left(\phi_{n}^{(1)}(\xi ; \alpha, \nu) ; \alpha, \nu\right) & =\xi, \quad \phi_{n}^{(1)}\left(G_{n}^{(1)}(t ; \alpha, \nu) ; \alpha, \nu\right)=t .
\end{array}
$$

Тогда $\forall(u, v)$ из единичного квадрата существует единственная копула $C_{G r}(u, v ; n, \alpha, \nu)$ : $[0,1]^{2} \rightarrow[0,1]$ такая, что

$$
C_{G r}(u, v ; n, \alpha, \nu)=\Upsilon_{n}\left(\phi_{n,(1)}(u ; \alpha, \nu), \phi_{n}^{(1)}(v ; \alpha, \nu) ; \alpha, \nu\right),
$$

где $\Upsilon_{n}\left(t_{1}, t_{2} ; \alpha, \nu\right)$ вычисляется согласно (11).

Замечание. В определении (57) предполагается, что $n \geq 3$, так как в этом случае частные распределения $G_{n,(1)}$ и $G_{n}^{(1)}$ являются непрерывными, то копула Граббса $C_{G r}$ определена единственным образом ([11], с. 18).

Теорема 3. Пусть $n \geq 3 u \Sigma_{n}=\left[0 \leq u \leq 1, \delta_{n}(u ; \alpha, \nu) \leq v \leq 1\right]$. Тогда $\forall(u, v) \in \Sigma_{n}$ копула Граббса совпадает с нижней границей Фреше-Хефдинга, т.е.

$$
C_{G r}(u, v ; n, \alpha, \nu)=u+v-1,
$$

əде

$$
\delta_{n}(u ; \alpha, \nu)=G_{n}^{(1)}\left(\theta_{n}^{+}\left(\phi_{n,(1)}(u ; \alpha, \nu)\right) ; \alpha, \nu\right),
$$

функции $G_{n}^{(1)}(\cdot)$ и $\theta_{n}^{+}(\cdot)$ вычисляются согласно (9) и (22). 
Доказательство. Обозначим

$$
u=G_{n,(1)}\left(t_{1} ; \alpha, \nu\right), \quad v=G_{n}^{(1)}\left(t_{2} ; \alpha, \nu\right) .
$$

Тогда $t_{1}=\phi_{n,(1)}(u ; \alpha, \nu)$ и $t_{2}=\phi_{n}^{(1)}(v ; \alpha, \nu)$. Применим формулу (42) с учетом (57) и $(60)$. В результате $\forall\left(t_{1}, t_{2}\right) \in \Xi_{n}$ получим

$$
C_{G r}(u, v ; n, \alpha, \nu)=G_{n,(1)}\left(\phi_{n,(1)}(u ; \alpha, \nu) ; \alpha, \nu\right)+G_{n}^{(1)}\left(\phi_{n}^{(1)}(v ; \alpha, \nu) ; \alpha, \nu\right)-1 .
$$

Учитывая $(55)$ и $(56), \forall\left(t_{1}, t_{2}\right) \in \Xi_{n}$ получим

$$
C_{G r}(u, v ; n, \alpha, \nu)=u+v-1 .
$$

Легко убедиться, что функции $t_{1}=\phi_{n,(1)}(u ; \alpha, \nu)$ и $t_{2}=\phi_{n}^{(1)}(v ; \alpha, \nu)$ осуществляют биективное отображение области $\Xi_{n}$ в плоскости $O t_{1} t_{2}$ на область $\Sigma_{n}$ в плоскости Ouv.

Действительно, воспользовавшись равенствами (55) и (56) и применив обозначения (60), получим

$$
\begin{gathered}
0 \leq u \leq 1, \text { если } 1 / \sqrt{n} \leq t_{1} \leq(n-1) / \sqrt{n}, \\
G_{n}^{(1)}\left(\theta_{n}^{+}\left(t_{1}\right) ; \alpha, \nu\right) \leq v \leq 1, \text { если } \theta_{n}^{+}\left(t_{1}\right) \leq t_{2} \leq(n-1) / \sqrt{n} \text { и } 1 / \sqrt{n} \leq t_{1} \leq(n-1) / \sqrt{n} .
\end{gathered}
$$

Заметим, что с учетом (59) $\forall t_{1} \in[1 / \sqrt{n},(n-1) / \sqrt{n}]$ можно записать

$$
G_{n}^{(1)}\left(\theta_{n}^{+}\left(t_{1}\right) ; \alpha, \nu\right)=G_{n}^{(1)}\left(\theta_{n}^{+}\left(\phi_{n,(1)}(u ; \alpha, \nu)\right) ; \alpha, \nu\right)=\delta_{n}(u ; \alpha, \nu) .
$$

Следовательно, если $\left(t_{1}, t_{2}\right) \in \Xi_{n}$, то $(u, v) \in \Sigma_{n}$. Поскольку любая копула может принимать только неотрицательные значения, то $\forall(u, v) \in \Sigma_{n}$ имеем $u+v-1 \geq 0$. Поэтому формула (61) эквивалентна (58).

Следствие 2. Участок кривой $v=\delta_{n}(u ; \alpha, \nu), 0 \leq u \leq 1$, лежит не ниже диагонали квадрата: $v=1-u, 0 \leq u \leq 1$, проходя через его вершины с координатами $(0,1)$ и $(1,0)$.

Следствие 3. В случае $n=3$ копула Граббса совпадает с нижней границей ФрешеХефдинга, т. е. $\forall(u, v) \in[0,1]^{2}$ имеем

$$
C_{G r}(u, v ; 3, \alpha, \nu)=\max \{u+v-1 ; 0\} .
$$

Доказательство. Заметим, что в случае $n=3$ совместная функция распределения статистик $T_{3,(1)}$ и $T_{3}^{(1)}$ примет вид [2]

$$
\Upsilon_{3}\left(t_{1}, t_{2} ; \alpha, \nu\right)= \begin{cases}0, & \left(t_{1}, t_{2}\right) \in \Delta_{3} \backslash \Xi_{3} ; \\ G_{3,(1)}\left(t_{1} ; \alpha, \nu\right)+G_{3}^{(1)}\left(t_{2} ; \alpha, \nu\right)-1, & \left(t_{1}, t_{2}\right) \in \Xi_{3},\end{cases}
$$

где

$\Delta_{3}=\left[1 / \sqrt{3} \leq t_{1} \leq 2 / \sqrt{3} ; 1 / \sqrt{3} \leq t_{2} \leq 2 / \sqrt{3}\right], \quad \Xi_{3}=\left[1 / \sqrt{3} \leq t_{1} \leq 2 / \sqrt{3}, \theta_{3}^{+}\left(t_{1}\right) \leq t_{2} \leq 2 / \sqrt{3}\right]$, функция $\theta_{3}^{+}(\cdot)$ определена согласно (22).

Отсюда, воспользовавшись (57) и (60), получим

$$
C_{G r}(u, v ; 3, \alpha, \nu)= \begin{cases}0, & (u, v) \in[0,1]^{2} \backslash \Sigma_{3} \\ u+v-1, & (u, v) \in \Sigma_{3}\end{cases}
$$

где $\Sigma_{3}=\left[0 \leq u \leq 1, \delta_{3}(u ; \alpha, \nu) \leq v \leq 1\right], \delta_{3}(u ; \alpha, \nu)=G_{3}^{(1)}\left(\theta_{3}^{+}\left(\phi_{3,(1)}(u ; \alpha, \nu)\right) ; \alpha, \nu\right)$.

Заметим, что равенство (63) примет вид (62), если граница области $\Sigma_{3}: v=\delta_{3}(u ; \alpha, \nu)$ совпадает с отрезком прямой $u+v=1,0 \leq u \leq 1$. Таким образом, необходимо проверить справедливость равенства

$$
u+\delta_{3}(u ; \alpha, \nu)=1, \text { если } 0 \leq u \leq 1 .
$$


Для этого, применив (60), (59), $\forall u \in[0,1]$ представим левую часть равенства (64) в виде

$$
u+\delta_{3}(u ; \alpha, \nu)=G_{3,(1)}\left(t_{1} ; \alpha, \nu\right)+G_{3}^{(1)}\left(\theta_{3}^{+}\left(t_{1}\right) ; \alpha, \nu\right), \text { если } 1 / \sqrt{3} \leq t_{1} \leq 2 / \sqrt{3} .
$$

Далее, учитывая (10) и (9), последнее равенство перепишем в виде

$$
L(u)=\int_{-t_{1}}^{2 / \sqrt{3}} F_{2}^{(1)}\left(\rho_{3}\left(t_{1},-x\right)\right) f_{\widetilde{T_{3}}}(x ; \alpha, \nu) d x+\int_{-\theta_{3}^{+}\left(t_{1}\right)}^{2 / \sqrt{3}} F_{2}^{(1)}\left(\rho_{3}\left(\theta_{3}^{+}\left(t_{1}\right),-x\right)\right) f_{\widetilde{T_{3}}}(x ;-\alpha, \nu) d x,
$$

где $L(u)=u+\delta_{3}(u ; \alpha, \nu)$ и $1 / \sqrt{3} \leq t_{1} \leq 2 / \sqrt{3}$.

Применив (15), (16) для случая $n=3, \forall t_{1} \in[1 / \sqrt{3}, 2 / \sqrt{3}]$ получим

$$
\begin{gathered}
\rho_{3}\left(t_{1},-x\right) \leq 1 / \sqrt{2}, \text { если } x \in\left[\theta_{3}^{-}\left(t_{1}\right), \theta_{3}^{+}\left(t_{1}\right)\right] ; \\
\rho_{3}\left(t_{1},-x\right)>1 / \sqrt{2}, \text { если } x \in\left(-2 / \sqrt{3}, \theta_{3}^{-}\left(t_{1}\right)\right) \cup\left(\theta_{3}^{+}\left(t_{1}\right), 2 / \sqrt{3}\right) ; \\
\rho_{3}\left(\theta_{3}^{+}\left(t_{1}\right),-x\right) \leq 1 / \sqrt{2}, \text { если } x \in\left[\theta_{3}^{-}\left(\theta_{3}^{+}\left(t_{1}\right)\right), \theta_{3}^{+}\left(\theta_{3}^{+}\left(t_{1}\right)\right)\right] ; \\
\rho_{3}\left(\theta_{3}^{+}\left(t_{1}\right),-x\right)>1 / \sqrt{2}, \text { если } x \in\left(-2 / \sqrt{3}, \theta_{3}^{-}\left(\theta_{3}^{+}\left(t_{1}\right)\right)\right) \cup\left(\theta_{3}^{+}\left(\theta_{3}^{+}\left(t_{1}\right)\right), 2 / \sqrt{3}\right) .
\end{gathered}
$$

Воспользовавшись подстановкой $t_{1}=\frac{2}{\sqrt{3}} \sin \zeta, \frac{\pi}{6} \leq \zeta \leq \frac{\pi}{2}$, нетрудно проверить справедливость утверждений

$$
\theta_{3}^{+}\left(\theta_{3}^{+}\left(t_{1}\right)\right)=t_{1} \text { и } \theta_{3}^{-}\left(\theta_{3}^{+}\left(t_{1}\right)\right)=-\theta_{3}^{-}\left(t_{1}\right), \text { если } 1 / \sqrt{3} \leq t_{1} \leq 2 / \sqrt{3} .
$$

Также легко убедиться, что $\forall t_{1} \in[1 / \sqrt{3}, 2 / \sqrt{3}]$ выполняется неравенство $-t_{1} \leq \theta_{3}^{-}\left(t_{1}\right) \leq$ $\theta_{3}^{+}\left(t_{1}\right)$. Поэтому, применив (1), с учетом (66)-(70) равенство (65) можно привести к виду

$$
\begin{aligned}
L(u)=\int_{-t_{1}}^{\theta_{3}^{-}\left(t_{1}\right)} f_{\widetilde{T}_{3}}(x ; \alpha, \nu) d x+\int_{\theta_{3}^{+}\left(t_{1}\right)}^{2 / \sqrt{3}} f_{\widetilde{T}_{3}}(x ; \alpha, \nu) d x+\int_{-\theta_{3}^{+}\left(t_{1}\right)}^{-\theta_{3}^{-}\left(t_{1}\right)} f_{\widetilde{T}_{3}}(-x ; \alpha, \nu) d x+ \\
+\int_{t_{1}}^{2 / \sqrt{3}} f_{\widetilde{T}_{3}}(-x ; \alpha, \nu) d x
\end{aligned}
$$

(здесь также учтено равенство (46) для случая $n=3$ ).

Используя переменную интегрирования $z=-x$, последнее равенство приведем к виду

$$
u+\delta_{3}(u ; \alpha, \nu)=\int_{-2 / \sqrt{3}}^{2 / \sqrt{3}} f_{\widetilde{T_{3}}}(x ; \alpha, \nu) d x .
$$

Учитывая для плотности $f_{\widetilde{T_{3}}}(\cdot)$ равенство (52), получим (64). Следовательно, (63) примет вид (62).

Замечание 2. Из следствия 3 вытекает, что при $n=3$ копула Граббса не зависит от параметров $\alpha$ и $\nu$.

Следствие 4. Для маргинальных функций распределения статистик $T_{3,(1)}$ и $T_{3}^{(1)}$ имеет место равенство

$$
G_{3,(1)}\left(T_{3,(1)} ; \alpha, \nu\right)+G_{3}^{(1)}\left(T_{3}^{(1)} ; \alpha, \nu\right)=1 .
$$

Доказательство. Возьмем равномерно распределенные на $[0,1]$ случайные величины $U$ и $V$, для которых

$$
U+V=1 \text {. }
$$

Тогда копула для совместного распределения $U$ и $V$ совпадает с нижней границей ФрешеХефдинга (см. доказательство теоремы 2 в [1]). Поэтому $P(U<u, V<v)=\max \{u+v-1 ; 0\}$. 
Рассмотрим случайные величины $T_{3,(1)}$ и $T_{3}^{(1)}$. В силу следствия 3 копула для совместного распределения $T_{3,(1)}$ и $T_{3}^{(1)}$ совпадает с нижней границей Фреше-Хефдинга. Учтем, что $\forall \alpha \in \mathbb{R}$ и $\nu>0$ каждая из случайных величин $G_{3,(1)}\left(T_{3,(1)} ; \alpha, \nu\right)$ и $G_{3}^{(1)}\left(T_{3}^{(1)} ; \alpha, \nu\right)$ имеет равномерное распределение на $[0,1]$. Применив $(71)$, получим

$$
G_{3,(1)}\left(T_{3,(1)} ; \alpha, \nu\right)+G_{3}^{(1)}\left(T_{3}^{(1)} ; \alpha, \nu\right)=1 .
$$

\section{3. ИССЛЕДОВАНИЕ ОБЛАСТИ $\Sigma_{n}$}

Согласно теореме 3 в области $\Sigma_{n}=\left[0 \leq u \leq 1, \delta_{n}(u ; \alpha, \nu) \leq v \leq 1\right]$ копула Граббса совпадает с нижней границей Фреше-Хефдинга. В случае, когда параметр $n=3$, область $\Sigma_{3}$ представляет собой прямоугольный треугольник с вершинами в точках $(0,1),(1,1)$ и $(1,0)$ (следствие 3$)$. Поэтому параметры $\alpha$ и $\nu$ не оказывают никакого влияния на форму области $\Sigma_{3}$. В случае $n>3$ область $\Sigma_{n}$ представляет собой криволинейный треугольник с вершинами в точках $(0,1),(1,1)$ и $(1,0)$, ограниченный прямыми $u=1, v=1$ и кривой $v=$ $\delta_{n}(u ; \alpha, \nu)$, расположенной не ниже диагонали единичного квадрата (следствие 2 ). Поэтому в случае $n>3$ параметры копулы могут оказывать влияние только на криволинейную границу $\delta_{n}(\cdot)$ области $\Sigma_{n}$.

Свойства функции $\delta_{n}(\cdot)$, необходимые в дальнейшем, содержит

Теорема 4. Для $n>2, \forall \alpha \in \mathbb{R}, \nu>0$ функция $v=\delta_{n}(u ;-\alpha, \nu)$ является обратной для функиии $v=\delta_{n}(u ; \alpha, \nu)$, m. е.

$$
u=\delta_{n}\left(\delta_{n}(u ; \alpha, \nu) ;-\alpha, \nu\right) ; \quad u=\delta_{n}\left(\delta_{n}(u ;-\alpha, \nu) ; \alpha, \nu\right),
$$

где $0 \leq u \leq 1$ и $\delta_{n}(\cdot)$ определена согласно (59).

Доказательство. Сначала заметим, что из (9) вытекает

$$
\phi_{n}^{(1)}(u ; \alpha, \nu)=\phi_{n,(1)}(u ;-\alpha, \nu), \quad \alpha \in \mathbb{R}, \quad \nu>0 .
$$

Для того, чтобы найти обратную функцию, разрешим уравнение

$$
v=\delta_{n}(u ; \alpha, \nu)
$$

относительно переменной $u$. С учетом (59) уравнение (73) можно привести к виду

$$
\phi_{n}^{(1)}(v ; \alpha, \nu)=\phi_{n}^{(1)}\left(G_{n}^{(1)}\left(\theta_{n}^{+}\left(\phi_{n,(1)}(u ; \alpha, \nu)\right) ; \alpha, \nu\right) ; \alpha, \nu\right) .
$$

Учитывая, что функция $\phi_{n}^{(1)}(\cdot)$ является обратной для $G_{n}^{(1)}(\cdot)($ см. $(56))$, получим

$$
\phi_{n}^{(1)}(v ; \alpha, \nu)=\theta_{n}^{+}\left(\phi_{n,(1)}(u ; \alpha, \nu)\right) .
$$

Далее с учетом (72) можно записать

$$
\phi_{n,(1)}(v ;-\alpha, \nu)=\theta_{n}^{+}\left(\phi_{n}^{(1)}(u ;-\alpha, \nu)\right) .
$$
Используя подстановку $\xi=\frac{n-1}{\sqrt{n}} \sin \varphi$, легко проверить, что $\forall \xi \in\left[\frac{1}{\sqrt{n}}, \frac{n-1}{\sqrt{n}}\right]$ имеет место
равенство

$$
\theta_{n}^{+}\left(\theta_{n}^{+}(\xi)\right)=\xi
$$

Применим функцию $\theta_{n}^{+}(\cdot)$ к обеим частям равенства $(74)$. С учетом (75) можно записать

$$
\theta_{n}^{+}\left(\phi_{n,(1)}(v ;-\alpha, \nu)\right)=\phi_{n}^{(1)}(u ;-\alpha, \nu) .
$$

Применим функцию $G_{n}^{(1)}(\cdot)$ к обеим частям последнего равенства. Воспользовавшись (56), находим

$$
u=G_{n}^{(1)}\left(\theta_{n}^{+}\left(\phi_{n,(1)}(v ;-\alpha, \nu)\right) ;-\alpha, \nu\right) .
$$


Откуда, учитывая (59), получим, что решение уравнения (73), т. е. функция, обратная для функции $v=\delta_{n}(u ; \alpha, \nu)$, имеет вид $u=\delta_{n}(v ;-\alpha, \nu)$.

Поменяем местами переменные $u$ и $v$, т. е. будем обозначать обратную функцию, как и прямую, буквой $v$. Тогда $\forall \alpha \in \mathbb{R}$ и $\nu>0$ функция $v=\delta_{n}(u ;-\alpha, \nu)$ является обратной для функции $v=\delta_{n}(u ; \alpha, \nu)$.

Для изучения влияния параметров $\alpha$ и $\nu$ на форму кривой $v=\delta_{n}(u ; \alpha, \nu)$ были проведены численные расчеты участка криволинейной границы $v=\delta_{n}(u ; \alpha, \nu)$, соединяющей точки $(0,1)$ и $(1,0)$. Для этого был разработан рекурсивный алгоритм вычисления значений функции $G_{n}^{(1)}(\cdot)$ по формуле $(9)$. Алгоритм был реализован в инструментальной среде Delphi. Для приближенного вычисления определенных интегралов в (9) и (1) использовалась квадратурная формула Гаусса, узлами которой являлись корни многочлена Лежандра восьмой степени $\left([12]\right.$, с. 343). Для вычисления значений плотности $f_{\widehat{T}_{n}}(\cdot)$ была использована рекурсивная формула, найденная в [8]. Контроль за точностью численного интегрирования осуществлялся с помощью двойного пересчета.

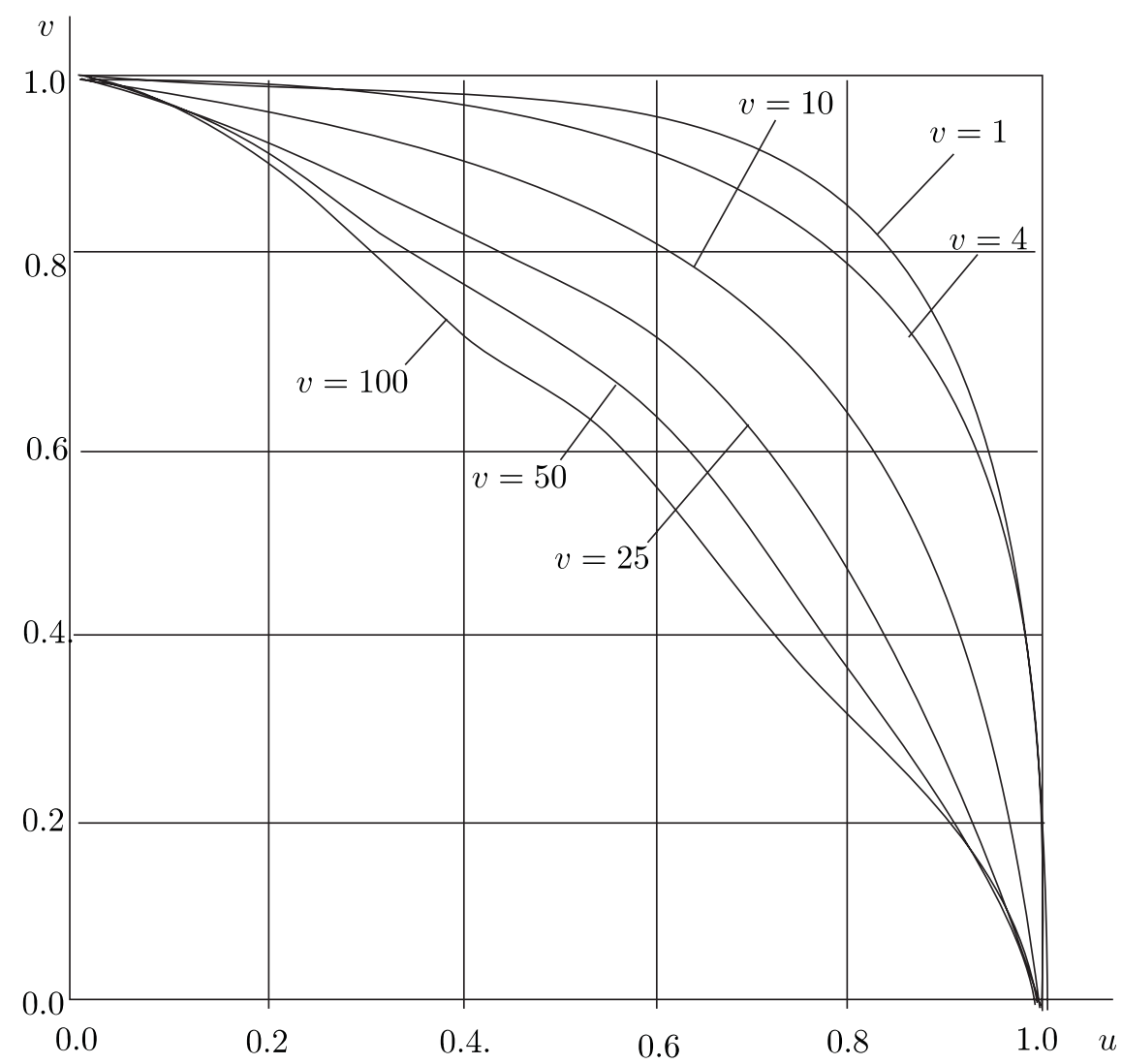

Рис. 1. Кривые $\delta_{7}(u ; 0, \nu)$ для значений $\nu$ от 1 до 100

Из теоремы 4 следует, что для значений аргумента $0 \leq u \leq 1$ кривая $v=\delta_{n}(u ;-\alpha, \nu)$ может быть получена отображением кривой $v=\delta_{n}(u ; \alpha, \nu)$ относительно прямой $v=u$. Кроме того, в случае, когда параметр $\alpha=0$, кривая $v=\delta_{n}(u ; \alpha, \nu)$ будет симметричной относительно прямой $v=u$. Поэтому численные расчеты участка криволинейной границы $\delta_{n}(u ; \alpha, \nu)$ были проведены для значений параметров $\alpha \geq 0, \nu>0$ и $n>3$. 
На рис. 1 приведены результаты численных расчетов границы $v=\delta_{n}(u ; \alpha, \nu)$ в случае, когда параметр $\alpha=0$ и $n=7$. Видно, что граница $v=\delta_{7}(u ; 0, \nu)$ симметрична относительно прямой $v=u$ и расположена не ниже диагонали единичного квадрата: $u+v=1,0 \leq u \leq 1$. С ростом параметра $\nu$ кривая $v=\delta_{7}(u ; 0, \nu)$ приближается к диагонали единичного квадрата. Аналогичным образом вела себя граница $v=\delta_{n}(u ; 0, \nu)$ и в случае $n \neq 7$.

На рис. 2 приведены результаты численных расчетов криволинейной границы в случае, когда параметр $\nu=1$ и $n=7$. Видно, как с ростом параметра $\alpha$ происходит движение кривой $\delta_{7}(u ; \alpha, 1)$ к диагонали единичного квадрата. Аналогичным образом вела себя граница $v=\delta_{n}(u ; \alpha, 1)$ и в случае $n \neq 7$. Из рис. 1,2 также видно, что параметры копулы $\alpha$ и $\nu$ могут оказывать различное влияние на криволинейную границу области $\Sigma_{n}$.

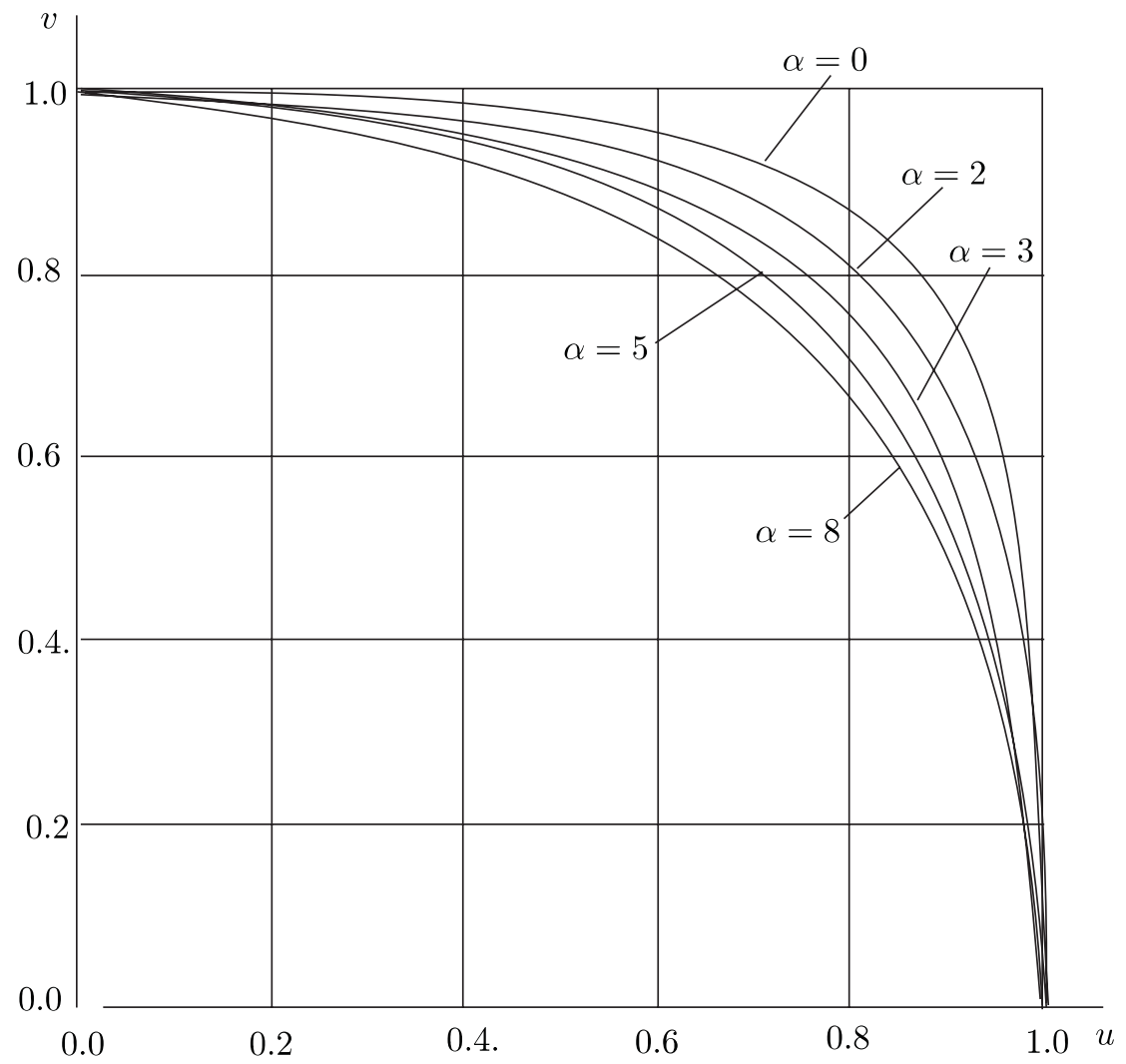

Рис. 2. Кривые $\delta_{7}(u ; \alpha, 1)$ для значений $\alpha$ от 0 до 8

Как известно, удобным инструментом для первичного анализа совместных распределений случайных переменных являются скаттерплоты. Скаттерплот представляет собой график сархивированных значений из копулы. Архивацию наблюдаемых значений случайных переменных производят посредством вычисления значений их кумулятивных функций распределения. Из существования области $\Sigma_{n}$, в которой копула Граббса совпадает с нижней границей Фреше-Хефдинга, следует, что $\forall(u, v) \in \Sigma_{n}$ плотность копулы $c_{G r}(u, v ; n, \alpha, \nu)=$ $\frac{\partial^{2} C_{G r}}{\partial u \partial v}(u, v ; n, \alpha, \nu)=0$. Поэтому для копулы $C_{G r}$ попадание точек скаттерплота в область $\Sigma_{n}$ невозможно. Следовательно, скаттерплоты из копулы Граббса всегда будут ограничены сверху границей $\delta_{n}(u ; \alpha, \nu)$. Примеры скаттерплотов из копулы Граббса, приведенные в [10] для случая $\alpha=0, \nu=1$ и $n \geq 5$, являются иллюстрацией указанного эффекта. 


\section{4. ЗАКЛЮЧЕНИЕ}

Рассмотрен случай, когда в нормальной выборке одно наблюдение (неизвестно какое по номеру) отличается от остальных величиной математического ожидания и дисперсии. Изучено совместное распределение односторонних статистик Граббса, т. е. стандартизированных минимума и максимума, вычисленных по нормальной выборке с выбросом.

Из совместного распределения стандартизированных минимума и максимума построена трехпараметрическая копула Граббса. Доказано существование области $\Sigma_{n}$, в которой копула Граббса совпадает с нижней границей Фреше-Хефдинга. Исследовано влияние параметров копулы на границы области $\Sigma_{n}$.

Показано, что скаттерплоты из копулы Граббса не могут пересекать область $\Sigma_{n}$. Это свойство можно учитывать при анализе скаттерплотов, построенных для реально существующих зависимостей. Если взаимное расположение точек скаттерплота позволяет предположить наличие некоторой верхней границы, выше которой точки скаттерплота не попадают, то для моделирования совместного распределения может быть применена копула Граббса.

Автор выражает искреннюю благодарность рецензенту за полезные замечания, позволившие улучшить содержание данной статьи.

\section{ЛИТЕРАТУРА}

[1] Фантаццини Д. Моделирование многомерных распределений с исполъзованием копула-функций. I, Прикл. эконометрика 22 (2), 98-134 (2011).

[2] Ширяева Л.К. О распределении статистик Граббса в случае нормалъной выборки с выбросом, Изв. вузов. Матем., № 4, 84-101 (2017).

[3] Pearson E.S., Chandra Secar C. The efficiency of statistical tools and a criterion for the rejection of outlying observations, Biometrika 28 (3-4), 308-320 (1936).

[4] Grubbs F.E. Sample criteria for testing outlying observations, Ann. Math. Statist. 21 (1), 27- -58 (1950).

[5] Barnett V., Lewis T. Outliers in statistical data (John Wiley \& Sons, Chichester, 1984).

[6] Zhang J., Keming Y. The null distribution of the likelihood-ratio test for one or two outliers in a normal sample, TEST 15 (1), 141-150 (2006).

[7] Ширяева Л.К. О нулевом и альтернативном распределении статистики критерия наибольшего по абсолютной величине нормированного отклонения, Изв. вузов. Матем., № 10, 62-78 (2014).

[8] Ширяева Л.К. Вычисление мер мощности критерия Граббса проверки на один выброс, Сиб. журн. индустриальной матем. 13 (4), 141-154 (2010).

[9] Ширяева Л.К. Использование специальных функиий Эрмита для исследования мощностных свойстө критерия Граббса, Вестн. Самарск. гос. техн. ун-та. Сер. Физ.-матем. науки 29 (4), 131-145 (2012).

[10] Ширяева Л.К. О хвостовой зависимости для копула-функции Граббса, Изв. вузов. Матем., № 12, 62-78 (2015).

[11] Nelsen R.B. An introduction to copulas. Lecture Notes in Statistics (Springer-Verlag, New York, 2006).

[12] Микеладзе Ш.Е. Численные методы математического анализа (ГИТТЛ, М., 1953).

\section{Людмила Константиновна Ширяева}

Самарский государственный экономический университет, ул. Советской Армии, д. 141, г. Самара, 443090, Россия,

e-mail: Shiryeva_LK@mail.ru 


\section{L.K. Shiryaeva}

\section{On three-parameter Grubbs copula-function}

Abstract. We study one-sided Grubbs's statistics for a normal sample, i. e. extreme studentized deviations of observations from the mean, computed from a normally distributed sample. We consider the case of the sample when there is one abnormal observation (outlier), unknown to what number according. The outlier differs from other observations in values of population mean and dispersion. We investigate the properties of the joint distribution of Grubbs's statistics. We prove the existence of a domain in which the joint distribution function of Grubbs's statistics is a linear function of their marginal distribution functions. We construct a three-parameter Grubbs's copula from the joint distribution of Grubbs's statistics. We prove the existence of a domain in which Grubbs's copula coincides with the Frechet-Hoeffding lower bound. We investigate the influence of the copulas parameters on the shape of this domain.

Keywords: one-sided Grubbs's statistics, standardized minimum and maximum, outlier, normal distribution, joint distribution function of standardized maximum and minimum, copula, FrechetHoeffding lower bound.

Lyudmila Konstantinovna Shiryaeva

Samara State Economic University,

141 Sovetskoi Armii str., Samara, 443090 Russia,

e-mail:Shiryeva_LK@mail.ru 